\title{
Orlov, Tarasov, and the Robinson sum rule*
}

\author{
Richard Talman \\ Cornell Laboratory of Elementary-Particle Physics, Cornell University, Ithaca, New York 14853, USA
}

Alexander W. Chao

Stanford Linear Accelerator Center, Stanford University, Stanford, California 94309, USA

(Received 15 December 2008; published 17 August 2009)

\begin{abstract}
A paper proving a result now commonly known as "Robinson sum rule" was published by Orlov and Tarasov \{J. Exp. Theor. Phys. 34, 651 (1958) [Sov. Phys. JETP 34, 339 (1958)] $\}$ at about the same time that Robinson himself published the result [Phys. Rev. 111, 373 (1958)]. We assigned ourselves the task of reviewing this work, as narrowly as possible, in hopes of understanding how it should be considered in view of the existing attribution. The chronology of the work is reviewed and the degree to which the two works were independent and have qualitatively different content is considered.
\end{abstract}

DOI: 10.1103/PhysRevSTAB.12.084901

PACS numbers: 61.80.Fe, 29.20.-c

\section{INTRODUCTION}

In accelerator physics there are many theories but few theorems, and few of those theorems are graced with the names of their discoverers. An example of a top named theorem would be "Liouville's theorem." The inventor's names are attached to their results initially as a mark of respect, but the eventual benefit of the practice is to abbreviate subsequent discussions. The mere mention of the name Liouville summons up an entire line of reasoning (including parts not actually due to Liouville).

Another example of named theorems, not much further down from that of Liouville's, is the theorem that is known as "Robinson's theorem" in the USA and in most of the rest of the world. This result was introduced by Robinson in the second half of the decade of the 1950s. Robinson was working on the design of the Cambridge Electron Accelerator (CEA), a $6 \mathrm{GeV}$ electron synchrotron jointly managed by Harvard and MIT.

To make progress at the outset, we first have to conclude that the term "theorem" is inappropriate here-the result is physics, not mathematics. The standards for mathematical proof and physical demonstration are so different that, even when used in discussion of physics, the term theorem conveys mathematical certainty rather than physical high likelihood. So the subject to be discussed and focused upon is the result commonly known as "Robinson's sum rule."

This small simplification alters the discussion noticeably. Issues such as motivation, scientific climate, applicability, and scientific impact seem more relevant than would be appropriate for a purely mathematical result. Also the less over-reaching term "sum rule" reserves appropriately, for numerous other authors, some of the credit for a sig-

\footnotetext{
${ }^{*}$ The web based version of this article at http://link.aps.org/ supplement/PRSTAB/v12/i8/e084901 is linked to separate auxiliary material. The web links in Refs. [1-3] lead to this auxiliary material.
}

nificant body of work to which the sum rule belongs as a piece.

At the same time as Robinson's work at CEA, at Yerevan in Armenia, similar research directions were being pursued. A plan was being developed to build a $3 \mathrm{GeV}$ (eventually $5 \mathrm{GeV}$ ) alternating-gradient electron accelerator. At that time Tarasov and Orlov, working together, and independent of Robinson, proved a similar result. Orlov had arrived at Yerevan only the previous year but he continued to collaborate with Tarasov who was at ITEP in Moscow.

Synchrotron radiation is responsible for a radiation damping mechanism in an electron accelerator. In terms of electron energy $\mathcal{E}_{e}$, the radiated power in bending magnets is proportional to $\mathcal{E}_{e}^{2}$. The radiation from an electron that temporarily has excess energy is above average. This tends to restore the electron's energy toward the average. The fact that the radiated power is also proportional to the square of the magnetic field complicates this tendency in accelerators (i.e. almost all accelerators) in which the magnetic field varies transversely in some or all of its elements.

There is also a damping mechanism for radial and vertical oscillations. The electrons pass through the $\mathrm{rf}$ cavity at that phase that, on the average, impulsively replenishes the energy lost to synchrotron radiation. With transverse momentum unaffected, but longitudinal momentum increased, there is a small net reduction in angle of trajectory. This effect provides damping to both radial and vertical betatron motions.

The radiated energy has to be made up by force supplied by the rf. Paying for this power seems like a small price to pay for the unmixed blessing of damping in otherwiseHamiltonian motion. However there is another cost; fluctuations in the radiation "heat" the beam. In the eventual equilibrium the heating is balanced by the "cooling" that is the subject of the result under discussion. This seems simple enough, but there is a further complication. Any 
single emission can represent either growth or damping of the oscillation amplitude of the particular particle radiating the photon. Any net damping can be correctly calculated only by correctly averaging over emissions, over electrons, and over magnetic lattice elements.

The result under discussion can be expressed as a sum rule; the sum of longitudinal, radial, and vertical damping rates is constant. This has been known as the Robinson sum rule. It provides a constraint on lattice design goals that is both powerful and easily applied. We know of no meaningful distinction between the Robinson theorem and the Robinson sum rule.

The purpose of this communication is to look into the history of this result, to identify its source, and if possible provide a recommended attribution. The situation is murkier than one would wish. As already mentioned, the significant publications all appeared within a year or so, and seem to be independent of each other. Also, because the Orlov-Tarasov method differs from Robinson's, the two works taken together have a significance going beyond either of the individual works.

It is not hard to figure out who did what. Much harder, is to assign relative importance to individual aspects of the overall result. Questions arise: Is the 3D result essentially more significant than the $2 \mathrm{D}$ result? Was the $2 \mathrm{D}$ result already accepted wisdom? Was one derivation stronger (i.e. fewer assumptions)? Are there ambiguities, or even disagreements, concerning terms in the sum rule? And there are other similar questions. We will address some of these questions. But, since the answers to questions like these are subjective, any attribution we might suggest can only be tentative at this point.

In what follows we first describe the climate of elementary-particle physics at the time, and then, first for Orlov-Tarasov, then for Robinson, describe their contributions. Following that we enumerate and discuss issues of attribution from various perspectives.

Robinson died in 1979 and Tarasov died a few years ago. Orlov is alive and well and living in Ithaca, New York, where he is Professor of Physics and Government at Cornell University.

\section{ATMOSPHERICS}

The year 1956 is a good time to start. A substantial first round of accelerator building after the Second World War had been completed. These were mainly "weak-focusing" machines. A timely accelerator conference was held that year: "The First CERN Symposium on High Energy Accelerators and Pion Physics" [4].

Simple scrutiny of the proceedings of that conference are helpful in avoiding some possible misconceptions. One possible misconception is that, because of the cold war, communication between east and west had been terminated. This may have been the case for individuals, but it was not true for the communities at large. For this conference the breakdown of home districts of the participants at the 1956 CERN conference was: Europe, 194; Soviet Union, 50; USA, 68; other, 7. These were not bureaucrats. They represented the cream of the worldwide crop of experimental elementary-particle physicists.

Bridging the ruptures of Europe had been a more important concern, especially at CERN, than was the cold war. Of course the iron curtain did constrain the movements of some individuals, especially from the Soviet Union. Orlov was prevented from attending the conference for political reasons, and there probably were others. Robinson also did not attend the conference. In any case, in that era, difficulties of language and geographical remoteness impeded communications more than did world politics.

Another thing to realize is that in the field of "elementary-particle physics," or "high energy physics," accelerators were the instruments that experimentalists built to do their experiments. In this conference the incipient splitting off of "accelerator physics" as a separate specialization was reflected only in the separation of the proceedings into two volumes, the first of which dealt primarily with accelerators, the second with particle experiments. In the present context this is relevant in only one respect. High energy physics was already "big science." Unlike more diverse fields, the field had already developed the tendency to identify relatively few issues as being "the important ones." Once a problem has been so identified, there is a friendly (or not) competition, among groups and individuals, to solve it. So Robinson, Orlov, and Tarasov were not laboring in obscurity. They were working on questions that were understood by and thought to be important by substantial segments of the worldwide community.

Already at that time there was a very respectable theory of the influence of synchrotron radiation on electron accelerators. Much of this understanding was due to Sands [5] but, naturally, many others had contributed. At the conference the understanding of the subject in Russia was reviewed at length by Kolomenskii and Lebedev [6]. Some of their results had probably been obtained independent of Sands.

At the CERN conference various "crazy" ideas were discussed. Examples were: colliding beams, based on storage rings by O'Neill and on FFAG by Kerst, electron cooling by Budker, and plasma acceleration by Fainberg, and separately by Veksler. Other ideas were also discussed, such as practical solutions to the problems associated with the successful completion of rings of the strong-focusing/ alternating-gradient/alternating-gradient synchrotron variety, commonly known as AGS, invented at Brookhaven a few years earlier.

To produce colliding beams, whether countercirculating in one ring or separately in two rings, two beams are 
required to collide in an intersection region at the center of a shared straight section. Nuclear cross sections are known to be so small that the beams are all but oblivious to each other. This is good in that the two beams can coexist while colliding; but also bad, in that the interaction rate is small. This makes it essential for the beams to circulate with small loss for millions, or actually billions, of turns before the rings would need to be refilled. This is very long compared to the tens of thousands of turns that beams are required to survive as they were being accelerated up to the energies at which they were extracted for fixed target experiments.

As mentioned earlier, this colliding beam possibility was just barely "in the air" at the time of the work being considered. As it happens, the sum rule we are discussing would eventually play a crucial role.

It is impossible to know, however, to what extent the possibility of colliding beams figured into the thinking of either Robinson or Orlov/Tarasov. Certainly they would have been aware of the great energy advantage to be obtained by having the laboratory frame and the center of mass frame coincide. But it seems that the first public statement of the plan to convert the CEA into a storage ring appeared in a 1965 lab report [7]. One has to assume that the sum rule came about primarily in the context of studying the stability of beams in electron synchrotrons. Such machines were being planned both at CEA and at Yerevan.

It seems to have been already known at that time that radial oscillations in an alternating-gradient ring would be antidamped. Taken at face value, this could impair the operation of an accelerator even during the relatively short time needed from bringing the electrons to full energy. As well as quantifying this, Robinson's paper [8] suggested what could be done to achieve long time stability of horizontal oscillations. His presentation, which amounted to generalizing the Sands theory to strong-focusing isomagnetic lattices, started by proving the result that has come to be known as his sum rule. This result helped to make the rest of his paper much more elementary and persuasive. In particular, shortly thereafter, he introduced his "Robinson wigglers" which helped CEA, by 1966, to successfully collide beams to study elementary-particle physics processes.

The motivation at Yerevan was similar. In a paper submitted for publication some six months before Robinson's publication, though not before Robinson's laboratory reports, Tarasov and Orlov published superficially similar results. They too discussed lattice modifications that could recover horizontal stability [9].

\section{THE ORLOV-TARASOV PAPER}

Orlov and Tarasov's paper [10] is focused quite narrowly on the description of radial-longitudinal coupled oscillations in an arbitrary ring. They set themselves the task of calculating the damping rates of (betatron) amplitudes $r$ and $z$ and longitudinal (synchrotron) amplitude $\phi$, as caused by synchrotron radiation. They seem to accept as already established the damping of betatron amplitude of an electron as it passes through the rf cavity. (In a published paper submitted eight months later, Orlov makes this argument far more clearly [11] while pointing out results contained in publications of others that he finds to be erroneous.)

Orlov and Tarasov used the Floquet formalism that was standard at the time in the U.S.S.R. (rather than the Courant-Snyder formalism). They permit arbitrary dependence on longitudinal coordinate $s$ of deviation from nominal, $H_{s}-H_{0}$, of the magnetic field. This generality will figure prominently in later discussion. Using the smooth approximation differential equations for longitudinal motion, and assuming (like everyone else) the vertical motion to be decoupled from the horizontal, they reduced the problem to two coupled, stochastic, second order differential equations for $r$ and $\phi$. After finding what is nowadays referred to as the dispersion function and averaging appropriately, they obtain the desired growth rate $\langle\dot{r}\rangle$.

They show that this formula reduces to a formula for a weak-focusing ring that is due to Sands. They also confirm the radial antidamping of alternating-gradient rings-a result that Tarasov and Orlov ascribe to Kolomenskii and Lebedev. They then calculate the phase damping decrement and show, for the special case of a weak-focusing ring, that this result also agrees with Sands.

As further confirmation of their results, they reduce the problem to one of finding the characteristic roots of their linearized equations. Though not showing the work in detail, they state that the same results are obtained.

After completing this calculation they state: "As was to be expected, the sum of the damping coefficients for radial and phase oscillations does not depend on the form of $\psi$ and $\left(H_{s}-H_{0}\right) / H_{0}$; i.e. it does not depend on the specific form of the radial-phase coupling." They had, by that time, proved the rule. On the other hand, since no identification was made, the question remains whether it was to be expected because it was mentioned in the earlier report by Robinson (which was referenced in [10]), or independently by the authors themselves. We have been unable to find a persuasive answer to this important question.

It is usually considered to be also important for the Robinson sum rule to include all three: horizontal, radial, and vertical damping rates. This aspect is not made explicit in the Tarasov-Orlov paper. But they discuss (and debunk) the possibility, using skew quadrupoles, of trading some vertical damping at the expense of an equal reduction of horizontal damping rate. This amounts to applying the sum rule in a special case.

Orlov and Tarasov then proceed (briefly) to describe the sort of accelerator sections that would be needed in order to restore stability to the radial oscillations in an alternatinggradient ring. 
In their final paragraph, while discussing "radially focusing magnets with zero or negative field," Orlov and Tarasov refer to an unpublished laboratory report, CEA-4 by Robinson, dated May 15, 1956 [1], and refer to "proposals of Livingston and Robinson."

Like Robinson, Orlov and Tarasov do not anticipate separated-function lattices in which some or all of the focusing is provided by pure quadrupoles (even though their formalism would have been applicable). Coming later, this development enables a controllable redistribution of damping from longitudinal to radial, from hindsight, in a rather straightforward manner.

\section{THE ROBINSON PAPER}

Unlike the paper of Orlov and Tarasov, which is succinct and in the Russian style, Robinson's sum rule result is contained in a paper [8] that, in the American style, discursively discusses the influence of synchrotron radiation on an electron ring. Roughly speaking, the paper updates an earlier paper of Sands to cover the newer accelerator designs. He clearly explains the damping of betatron oscillations in an rf cavity, but he does not make it clear by reference to previous publications whether he considers this to be original work. Perhaps this is an oversight? Perhaps he refrains from referring to his own earlier work? Similar comments can be made about other topics contained in the paper. None of these bear on the issue at hand.

Robinson's proof of the sum rule is clearly explained in his paper and has been revised but little in subsequent lecture notes and textbooks. In Appendix A an abbreviated line of reasoning is given that is intended to show why a "damping sum rule" is to be expected.

Robinson (like Orlov/Tarasov) fleshes out a chain of reasoning like this. Irrespective of injection conditions, if all modes are stable, an equilibrium is eventually reached in which individual damping rates and excitation rates of every mode exactly cancel. For both damping and excitation the total rates are directly calculable. The sum rule disciplines lattice design, typically by showing how excess longitudinal stability can be sacrificed to produce horizontal stability.

When applied to combined-function lattices, because of excess energy damping, the sum rule makes the antidamping of radial oscillations inevitable. Furthermore, the sum rule suggested that revised lattice design could redistribute the damping. Later Robinson figured out one way of doing this to restore stability-by installing "damping wigglers." They were installed in 1966. This tour de force of accelerator science, one supposes, contributed to the attaching of Robinson's name to the sum rule. There was further recognition-the wigglers are known as Robinson wigglers.

Almost as useful as the sum rule itself, Robinson's paper also included a consistent collection of results that had been obtained previously by other accelerator scientists.
Now, as in the case of Liouville's theorem, the mere mention of the name "Robinson" summons up not just the sum rule itself, but a whole realm of understanding.

The sum rule applies to normal mode damping rates. His demonstration shows this for an arbitrary lattice. In this sense Robinson's result constituted a valid whole.

Nevertheless Robinson's paper [8] has significant limitations. When he himself applies the sum rule (to infer horizontal damping rate from the longitudinal rate) he does not, in fact, use normal mode rates. Also his subsequent calculations based on the sum rule are restricted to isomagnetic lattices. This prevents its use in important cases, the most important of which (because of its eventual universal adoption) is the separated-function case.

\section{ATTRIBUTION ISSUES}

We make the following observations.

1. A possibly helpful distinction to be drawn is that the Robinson approach to establishing the sum rule is deductive while the Orlov-Terasov approach is constructive. Simplifying a bit, let us say that the equality of two quantities, $A$ and $B$, is to be established. The deductive approach proves $A=B$, irrespective of the lattice details that influence $A$ and $B$, but without calculating either $A$ or $B$ explicitly. In the constructive approach, formulas are exhibited for $A$ and $B$, and shown to be equal, term by term, irrespective of lattice details.

As regards demonstrating the equality we have no reason to favor either approach over the other. But there are peripheral issues. The deductive approach is briefer and more elementary and, hence, it seems fair to say, more elegant. But it is also more limited. A dividend of the constructive approach is the actual calculation of the damping rates that are the subject of the sum rule.

2. During 1955-1958 the effect of synchrotron radiation on electron beams was being studied independently by several people. It was a hot topic of research triggered by the electron circular accelerators at CEA and Yerevan among others. Several people were busy calculating the damping rates. The race was mainly who could calculate them correctly. The most urgent problem faced by these accelerator physicists was the fact that it was found that, for alternating-gradient circular accelerators designed and built at that time using combined-function magnets, the horizontal betatron motion had radiation antidamping, and these accelerators might be inadequate as synchrotrons and would be fundamentally unsatisfactory as storage rings.

Also well known at the time were quantum excitation calculations, and the conceptual basis for determining equilibrium beam emittances as a balance between quantum excitation and radiation damping. Basically, all basic ingredients as we know them were on the table, except the sum rule. The approach had been to calculate the damping rates without realizing the hidden sum rule. This observation is more in line with an argument that the attribution 
really should belong to the people who first realized the existence of the sum rule.

3. The first breakthrough came when Robinson suspected the existence of the sum rule in the unpublished lab report [1], dated May 15, 1956. It stated that "...The sum of the damping rates is then $\rho_{T}=2 V_{0} f_{0} / E_{0} \ldots$ This total damping rate seems to be a fundamental characteristic of the nature of the radiation loss, and the radiofrequency system required to supply this loss ...." From this observation, Robinson then suggested two possible ways to stabilize the horizontal betatron motion: (a) Use combined-function wigglers, and (b) use skew quadrupoles to couple the horizontal and vertical betatron motions while making $\nu_{x} \approx \nu_{y}$. As it happens, neither of these suggestions is found to be very effective.

4. Robinson then gave a proof of the sum rule in an unpublished internal laboratory report [2], dated April 10, 1957. An updated version then appeared in another unpublished laboratory report [3], dated January 6, 1958. In [3], Robinson combined and expanded Refs. [1,2]. This report [3] was then sent for journal publication [8], submitted March 17, 1958, revised June 2, 1958, and published July 15, 1958. In [8,3], Robinson referenced a list of papers and reports $[5,6,12-14]$ when speaking of past work on the calculation of radiation damping rates. No reference was made of [10] by Orlov and Tarasov.

5. In parallel to the developments led by Robinson in the USA, there was a separate line of research led by Orlov and Tarasov in the Soviet Union [10], submitted on September 6, 1957, published March 1958. An English translation was published September 1958. Using a formalism different from Robinson, and for a fully general lattice, they calculated the horizontal betatron and the synchrotron radiation damping rates, and exhibited the sum rule for these two dimensions. They also alluded to a sum rule between horizontal betatron and vertical betatron motions and explained why skew quadrupole coupling will not solve the horizontal instability problem because the sum of horizontal betatron and vertical betatron motions would be zero, i.e., no net damping. They ascribe the intentional coupling proposal (somewhat derisively) to Kolomenskii [15], apparently unaware of the suggestions also having been made by Robinson.

6. References made in [10], when referring to past work on radiation damping, included $[5,6,1,15,16]$. In particular, Robinson's result [1] and the proposed possible existence of a sum rule seems to have been known to the authors. Paper [10] set out to prove the sum rule using its own approach. The timing of [10] was after Robinson's result [2]. So [10] was not the first proof of the sum rule.

Arguments, however, reasonably persist concerning the following considerations: (a) result [2] was unpublished and, therefore, not necessarily recognized; (b) the discussion accompanying [2] is quite specialized (isomagnetic assumption); and (c) the mathematics accompanying [2] is (arguably) flawed in its averaging assumptions. We give our opinions of considerations (a) priority, (b) generality, and (c) validity, next.

7. In the modern era, with near instant worldwide communication, consideration (a) would favor Robinson. Robinson proposed the sum rule [1] and proved it [2] before Orlov and Tarasov [10]. Also, after having written [1], Robinson could have been expected to continue his work. It would have been natural for Orlov and Tarasov to follow up on what Robinson might have done subsequently on the topic before they sent their paper to be published. They might have found report [2] and might then have revised their paper to emphasize the ways in which their treatment was more powerful. Nowadays, therefore, Orlov and Tarasov might be criticized for lack of research of existing reports (a fault not unknown among modern researchers) in preparing paper [10]. All this is mitigated to a large extent by problems of language, geography, cold war, and the generally inferior communications available in 1957. We are unable to assess the impact of these differences persuasively.

Considerations (b) and (c) are more subject to objective discussion. The Orlov-Tarasov formalism, unlike the Robinson formalism, provides formulas applicable, for example, to separated-function lattices. Since separatedfunction lattices constitute the most important application of the topic under discussion, this extra generality is essential. Consideration (c) is more arcane, and any attempt to clarify it is likely to be controversial. The reader wishing to investigate this further could profitably begin with an article by Kolomenskii and Lebedev [17]. Writing in 1957, they are familiar with the original papers of both Orlov/Tarasov and Robinson. They state that "strong focusing was first considered by Robinson and later, in a generalized form, by Orlov and Tarasov." Unfortunately they do not express an opinion as to the relative theoretical validity of the two approaches.

For the actual evaluation of terms appearing in the sum rule it is very important to resolve all the theoretical issues we have labeled as (c). And they are not at all trivial. Especially important is the requirement that the individual terms in the sum rule correspond to normal modes of oscillation. When the terms in the sum are regarded as experimentally measured numbers this is automatic, as it is the normal modes that make themselves visible to the experimenter. But the correct treatment of normal mode coordinates is theoretically difficult.

Nevertheless we have not analyzed the original papers critically in this respect of theoretical validity. We have two excuses for this. One excuse is that argument (c) seems (to us) to be far less important than (b). The (b) generality provided only by the Orlov/Tarasov paper has been essential for the application of the sum rule to all subsequent colliding beam applications. So derating the significance of (c) seems unlikely to influence any overall attribution of credit. 
Our other excuse is that issues of theoretical validity relate to the application of the sum rule, not to the introduction and proof of the sum rule itself. Even if Robinson's use of the sum rule can be criticized, the proof itself is completely general and valid.

8. There is another distinction between the two results. Stochasticity seems to play no role in the Robinson derivation. Demonstration of the Orlov-Tarasov sum rule, on the other hand, starts from differential equations that are explicitly stochastic. Proof of the sum rule depends on the correct solution of these equation (by averaging). This difference is fundamental enough to make it clear that the sum rules proved are not exactly equivalent. The character of the difference is reminiscent of the fluctuation/ dissipation theorem of nonequilibrium statistical physics [18].

\section{CONCLUSION}

While attempting to assign credit, it is essential to establish what result or results are in question. It can be argued that focusing on the name of the sum rule does not represent the actual history nor does it illuminate the significance of work that may actually have been more important than the sum rule itself.

In its narrowest sense the sum rule relates experimentally observable quantities. Normal mode oscillations manifest themselves, for example, as peaks on a spectrum analyzer. Over time each mode damps by a measurable amount. According to the sum rule the sum of these rates is equal to another unambiguously measurable quantity.

Robinson was apparently the first to suspect the existence of the sum rule in the accelerator context and the first to prove it. Furthermore his proof is valid for general accelerator lattices. History has therefore been fair in assigning (at least) Robinson's name to the sum rule.

But this definition of the work seems overly narrow. For example, it reduces the result to being a straightforward application of the long-established, LiouvilleOstrogradsky result (which is sketched out in Appendix A).

The issue then is whether history has been unfair in not adding the names of Orlov and Tarasov. With a slight offset in time, following immediately and independently, and using a completely different formalism, they obtained similar results.

Neither Robinson nor Orlov/Tarasov emphasized the importance of the sum rule in the narrow sense described so far. (Neither did they refer to the result as a theorem.) They both regarded the rule as a means toward a practical end - the evaluation (and eventual adjustment) of individual damping rates. In other words, for both groups, it was the application of the sum rule to describing beam evolution in accelerators that was essential.

As regards the application of the sum rule, the Orlov/ Tarasov approach was more powerful than Robinson's. Since their proof was constructive, it explicitly contained the results for which the sum rule was intended to be used. Unlike Robinson's subsequent application of the sum rule (in the same paper), which used the isomagnetic limitation, the Orlov/Tarasov treatment is applicable to arbitrary accelerator lattices, such as separated-function lattices. Also, by explicitly working with normal modes, the Orlov/ Tarasov approach includes strongly coupled cases.

We have reconstructed the history as faithfully as we are able. We do raise the issue of, and leave it to individuals and to the community to decide, whether the results we have been discussing should be renamed as the RobinsonOrlov-Tarasov sum rule.

\section{ACKNOWLEDGMENTS}

This work was supported by National Science Foundation Contract No. PHY-9809799 and Department of Energy Contract No. DE-AC02-76SF00515.

\section{APPENDIX A: WHY A SUM RULE SHOULD BE EXPECTED}

For one-dimensional motion one is accustomed to introducing cosinelike $C(t)$ and sinelike $S(t)$ orbits satisfying initial conditions $[C(0), \dot{C}(0)]=(1,0)$ and $[S(0), \dot{S}(0)]=$ $(0,1)$. In terms of these functions a "transfer matrix" $\mathbf{M}(t)$ governs evolution of position vector $\mathbf{X}(t)$ according to

$$
\mathbf{X}(t)=\mathbf{M}(t) \mathbf{X}(0) \equiv\left(\begin{array}{cc}
C(t) & \dot{C}(t) \\
S(t) & \dot{S}(t)
\end{array}\right) \mathbf{X}(0) .
$$

The determinant $\operatorname{det} \mathbf{M}(t)$, known as the Wronskian, has two important properties: in Hamiltonian lattice theory its constancy follows from the Newton force law, and its value is the oriented area of the phase space parallelogram with edges defined by $C(t)$ and $S(t)$. Taken together these facts assure the constancy of phase space densities.

In his textbook on differential equations, Arnold [19] generalizes this argument in ways we need for nonHamiltonian lattice theory in three dimensions. The quantity $W(t) \equiv \operatorname{det} \mathbf{M}(t)$, still called the Wronskian, is formed from the six phase space coordinates of any six independent orbits. $\mathbf{M}(0)$ could, for example, be block diagonal with a $2 \times 2$ block as in Eq. (A1) for each of three orthogonal spatial dimensions. For $t>0$ the block diagonality will, in general, be lost, for example, because of coupling. But $W(t)$ can still be interpreted as the oriented (now) hypervolume of the phase space region defined at time $t$ by the assumed orbits.

Arnold proves the following Liouville theorem, sometimes ascribed also to Ostrogradsky. The equation governing time evolution of $\mathbf{M}(t)$ is

$$
\frac{d \mathbf{M}}{d t}=\mathbf{A}(\mathbf{t}) \mathbf{M}
$$

which is the Poincare linearized equation for small variations from the nominal orbit. Finding the coefficients of $\mathbf{A}$ 
is straightforward. The equation's approximate solution, valid for small $t$, is

$$
\mathbf{M}(t)=\exp [t \mathbf{A}(\mathbf{0})+\cdots] \mathbf{M}(0) .
$$

Taking determinants and using the rule for the product of determinants as well as a formula for the determinant of an exponential that is also due to Liouville, yields, for small $t$,

$$
W(t)=\exp [t \operatorname{tr} \mathbf{A}(0)] W(0) .
$$

where $\operatorname{tr} \mathbf{A}(\mathbf{t})$ is the sum of diagonal elements of $\mathbf{A}(t)$. Then $W(t)$ satisfies

$$
\frac{1}{W} \frac{d W}{d t}=\operatorname{tr} \mathbf{A}(t)
$$

In spite of the small $t$ approximation used in the derivation, this formula is valid for arbitrary $t$ since the time origin was arbitrary. This equation can be solved in closed form, but for present purposes it is enough to note that $\operatorname{tr} \mathbf{A}(\mathbf{t})$ is the fractional change of phase space volume per unit time.

This formula can be applied to our lattice that has been caused by synchrotron radiation to be slightly nonHamiltonian. Suppose that it is legitimate to assume that A can be block diagonalized into horizontal, vertical, and longitudinal $2 \times 2$ matrix blocks. On the right-hand side of the equation, because of the trace operation, the growth rate is the sum of the separate growth rates. In case block diagonization into $r, y$ and $z$ blocks is not applicable, e.g., when couplings are too strong, then the sum refers to a sum over the three eigenmodes, and the sum rule still applies.

[1] Kenneth W. Robinson, CEA-4, 1956 (unpublished).
[2] K. W. Robinson, CEA-28, 1957 (unpublished).

[3] Kenneth W. Robinson CEA-44, 1958 (unpublished).

[4] E. Regenstreif, CERN Symposium on High Energy Accelerators and Pion Physics, Geneva, 1956, CERN 56-25.

[5] M. Sands, Phys. Rev. 97, 470 (1955).

[6] A. A Kolomenskii and A. N. Lebedev, in CERN Symposium on High Energy Accelerators and Pion Physics, Geneva, 1956, p. 447.

[7] K. Robinson and G. Voss, CEA-TM-149, 1965 (unpublished). This and other CEA reports can be obtained from the MIT Libraries Document Services, Cambridge, MA.

[8] K. Robinson, Phys. Rev. 111, 373 (1958).

[9] As an aside, it can be noted that no corrective elements were included in the Yerevan ring and, in spite of its incipient horizontal instability, the ring went on to function satisfactorily as a source of beams for fixed target experiments.

[10] Iu. F. Orlov and E. K. Tarasov, J. Exp. Theor. Phys. 34, 651 (1958) [Sov. Phys. JETP 34, 3 (1958)].

[11] I. Orlov, J. Exp. Theor. Phys. 35, 525 (1958).

[12] D. Bohm and L. Foldy, Phys. Rev. 70, 249 (1946).

[13] J. Henry, Phys. Rev. 106, 1057 (1957).

[14] K. Robinson and D. Ritson, CAP Report 14 (1955) (unpublished).

[15] A. A. Kolomenskii, Doctoral dissertation, Physics Institute, Academy of Sciences, U.S.S.R., 1957.

[16] A. A. Sokolov and I. M. Ternov, Dokl. Akad. Nauk SSSR 97, 823 (1954).

[17] A. Kolomenskii and A. Lebedev, At. Energ. 5, 554 (1958) [J. Nucl. Energy, Part C, Plasma Phys. 1, 139 (1960)].

[18] L.D. Landau and E. M. Lifshitz, Statistical Physics (Pergamon Press, Oxford, 1985), 3rd ed., Part 1, Chap. 12.

[19] V. I. Arnold and Yu. S. Il'yashenko, Ordinary Differential Equations, in Ordinary Differential Equations and Smooth Dynamical Systems (Springer-Verlag, Berlin, 1997). 